\title{
Self-healing cement materials - microscopic techniques
}

\author{
Marta Dudek \\ Chair of Building Materials Engineering; Faculty of Civil Engineering; \\ Cracow University of Technology; Warszawska St. 24, 31-155 Cracow, Poland; \\ marta.dudek@pk.edu.pl (iD)0000-0003-0658-6922
}

\begin{abstract}
The article presents a general classification of intelligent materials with self-healing (self-repairing) properties, focusing on self-healing cementitious materials. The purpose of the paper is to describe the prospects of two of the most popular micro-observation techniques, i.e. with the use of an optical and scanning electron microscope. In addition, it describes the advantages of using a tensile stage mounted in the microscope chamber for testing self-healing materials. The advantages and disadvantages of these devices have been characterized, and the results of preliminary research have been provided. The tests include the optical microscopy and scanning electron microscopy observations of the microstructure of cracks before and after the process of healing. They were carried out using ZEISS Discovery V20 optical microscope and ZEISS EVO-MA 10 scanning electron microscope on mortar samples modified with macro capsules filled with polymer. In addition to observations, chemical analysis was performed with the use of an EDS detector. The microscopic observations and chemical analyses provide the basis for assessing the effectiveness of the self-healing process, showing that the crack has been healed. Moreover, the preliminary results of the tests of micro-mechanical properties, carried out with the use of a tensile stage, have been described. The problems of using this research technique are also listed. This study shows the usefulness of this kind of tests for microcapsules for self-healing materials.
\end{abstract}

Keywords: optical microscopy, scanning electron microscopy, tensile stage, self-healing cementitious materials, micro-mechanical properties

\section{Introduction}

Intelligent materials are the subjects of many scientific research conducted in various branches of science. According to Takagi [1] "intelligent materials" are materials which are able to react (respond) to external impulses by significantly changing its own properties and obtain expected and effective reaction to such impulses, whereas "smart materials" only have a predictable effect of changing the properties under external impulses. The group of intelligent materials comprises of materials with an ability to change colour, emit light, change shape 
and size (also with shape-memory properties), change temperature and density, self-group, self-heal and self-repair.

The research conducted all over the world on self-healing (self-repairing) materials were inspired by the nature, and especially self-healing processes which occur e.g. in human body. For example, injured skin or broken bone has the ability to heal without human intervention. Bearing this in mind, scientists were striving to develop materials with the capability of self-healing in case of damage. These materials are also known as intelligent materials, as they can sense changes, process information and respond to these changes [2]. According to Zwaag [3], self-healing materials are those materials which have negative damage rate at one or several stages of use. It means that damages heal themselves, and as a result, over time there are fewer damages than there were at the beginning. The effectiveness of this process depends on the speed of damage formation and the speed of self-healing. Polymers are the materials which have best self-healing properties. Then, there are ceramic materials, especially cement materials. The most problematic group, in the context of these abilities, are metals [4].

The aim of the self-healing (self-repairing) process of cement materials is to completely or partially recover their original properties or contain destructive processes (for example the propagation of crack) [5]. Self-healing cement materials, due to their fragile nature, are mainly destined to close the crack.

According to RILEM Technical Committee [5], self-healing involves all the processes leading to the recovery of the properties. They are divided into two basic groups:

- Autogenous: a self-healing process is autogenous when it concerns components of materials which have been used to form that material (they were not added for the purpose of obtaining self-healing properties).

- Autonomous: a self-healing process is autonomous when it uses components which have been added for the purpose of obtaining self-healing properties. They can be for example mineral additives, capsules filled with self-healing materials, and many others.

The autogenous self-healing process of concrete consists in filling and sealing cracks caused by chemical reactions with non-hydrated concrete mixture components and carbonation reactions, which are natural reactions for setting of the cement bond. From this point of view, cementitious materials, by their nature, have high self-healing potential. The reason is the hydration process of cement components, which lasts for years, and most of all, presence of non-hydrated or partially hydrated cement grains. When the concrete cracks, these grains react with the moisture, forming additional phases of concrete sealing. However, in the process of autonomous healing, cracks are closed by hydration of mineral substances that were added to the materials for the purpose of obtaining such characteristic. They have pozzolanic and expansive properties, after having reacted with water, and they trigger new products of hydration. On the other hand, activated repair is based on healing properties of components which were added during the production process, for example capsules filled with polymer resin or with bacteria.

\section{Materials and Methods}

Depending on the aim of self-healing (self-repairing) process, there are different techniques used to verify the effectiveness of that process. If the purpose of the process of recovery of the original properties is to improve the resistance to external environment, the tests that allow to assess the permeability of material are used. Whereas, in case of mechanical 
recovery, strength tests are preferable. However, microscopic tests are very important in all of the processes. Their aim is usually to measure occurring cracks, assess the process of cracks propagation over time, and also to assess the self-healing process of the material.

\subsection{Optical microscopy}

An optical microscope is used to magnify the image, due to its construction consisting of a system of lenses, through which a beam of light is sent. As a result, an enlarged and inverted image is received. The research with the use of optical microscopy is one of the ways to assess the width of the crack during the process of sealing in self-healing materials. This technique is cost-effective and easy to handle. It provides, in comparison to scanning electron microscopy, the possibility of colourful sample observation, which helps to visualize the process of crack sealing. It makes it also possible to observe samples of much greater size. What is more, it provides bigger field of observation, which makes it easier to analyse cracks, the structure of materials, pores and aggregates. In this case, to obtain a good quality of image, a user can operate parameters such as: work distance, magnification, lighting, filters, and distribution of optical elements [5]. Numerous publications indicate that the use of this device is prevalent amongst researchers $[6,7]$. Many of them have been using optical microscopes for assessing the effectiveness of sealing cracks in mortars with biological substances closed in lightweight aggregates, and also for the observation of the influence of powdered materials closed in self-healing capsules.

\subsection{Scanning electron microscopy}

The principle of image formation in scanning electron microscopy is different than in optical microscopy. This technique does not use light beams; the observed sample is scanned by focused high-energy electron beam generated with the use of the electron gun. In this process, electrons interact with atoms in the sample, and the tested material emits a variety of signals recorded by detectors. These signals are secondary electrons, backscattered electrons, and characteristic X-rays. Subsequently, the detectors process them into an image. Secondary electrons are responsible for creating the image with information about the topography of the sample; backscattered electrons, on the other hand, give better phase contrast, while the characteristic X-rays allow for the elemental analysis of the composition. SEM yields much greater magnifications (several to dozen of thousands of times) and at the same time, it maintains the depth of focus and good resolution. Its main disadvantage, in comparison to the optical microscope, is the fact that the image is in shades of grey, which necessitates the use of small and very small samples, which very often also requires a proper preparation, and provides a smaller view. However, it enables more detailed analysis of the material, especially if there is a need to see the elements invisible to the naked eye. It allows also to make a chemical elemental analysis during sample observation by using special detectors. The quality of images is impacted among the others by pressure in the chamber, work distance, material contamination, spot size, scanning speed and accelerate voltage [5].

Due to the size of magnification, scanning electron microscopy is more often used in the research concerning self-healing materials than optical microscopy. The authors of the publication [9] about self-healing concretes modified with capsules used SEM to assess the appearance of capsules and their micro-morphology [10]. In particular, they determined their diameter and layer thickness. The researchers compared the effect of calcium-nitrate 
micro-capsules on further concrete strength by modification of the encapsulation method, and they assessed how it affects strength reduction. With this technique, it is possible to estimate the self-healing capabilities of cementitious composites [11], not only by observation of cracks and the area after their sealing, but also by assessment of the chemical elemental composition of healing products with the use of EDX method. In this advanced technique, it is also possible to monitor the process of leaking of the healing substance from capsules, to observe sealing process, and to determine what affects its speed [10].

\subsection{Tensile stage mounted in scanning electron microscope}

The DEBEN MICROTEST 200N tensile stage is made to be mounted in the scanning electron microscope chamber (Figure 1). It gives the possibility to determine the tensile, compressive and fatigue strength during the simultaneous microstructure observations in SEM. The working range of the device is $200 \mathrm{~N}$; it works at a speed from $0.1 \mathrm{~mm} / \mathrm{min}$ to $1.5 \mathrm{~mm} /$ min. During the test, a graph is drawn which shows the influence of force on elongation or time. It can be used to assign micro-mechanical properties.
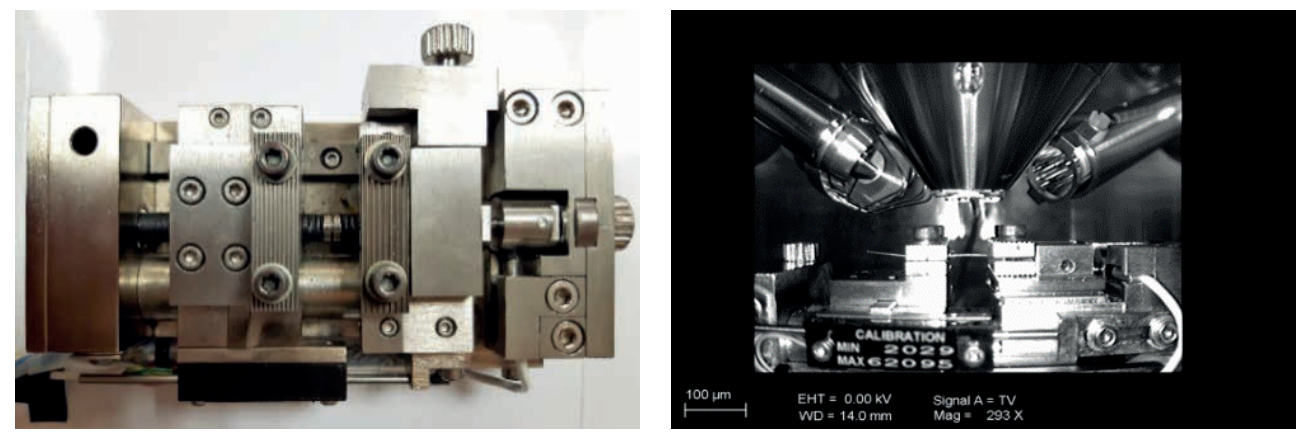

Fig. 1. Tensile stage - general view (on the left), scanning electron microscope chamber with mounted tensile stage (on the right). Source: author's own study

\section{Results and discussion}

\subsection{The results of optical microscopy}

This research was performed by means of a ZEISS Discovery V20 optical microscope with magnification up to $345 \mathrm{x}$. The tool has the option to change parameters such as magnification, focus, contrast, and brightness during observations. Luminous methods and contrasts are achieved by LEDs or cold light.

Sample results of the observation of cracks after the healing process are presented in the Figure 2. For the study mortars beams were used $\left(40 \times 40 \times 160 \mathrm{~mm}^{3}\right)$, modified with glass capsules filled with polymer. After initiating a crack made with 3-point bending test, the capsule burst, and the healing agent filled the crack. The image on the left shows a sample of the cut mortar with the capsule, while the image on the right shows the bottom of the beam along the crack, where the polymer flows. 

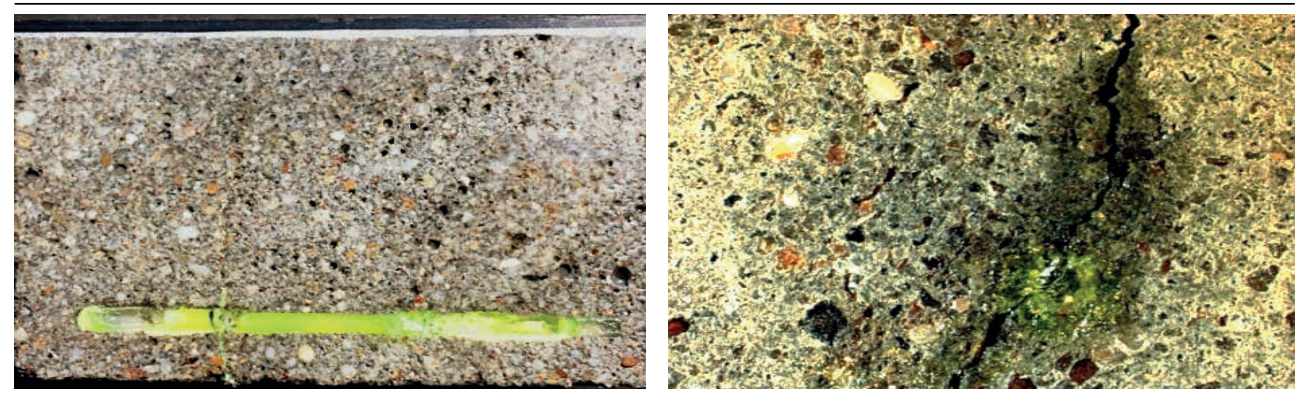

Fig. 2. On the left: image of a sample of the cement mortar with capsule filled with polymer viewed with optical microscope Zeiss Discovery V20. On the right: crack after healing. Yellow colour indicates the place where polymer leaked from the capsule in the place where the crack occurred. The source: author's own study

\subsection{The results of electron microscopy}

Microstructure observations were carried out on the same samples as with the use of optical microscope (Figure 3) by the author of the paper. For the test, a ZEISS EVO-MA 10 scanning electron microscope was used, equipped with SE, VPSE and BSD detectors, as well as with the EDS Bruker XFLASH 6/30 detector, that facilitates chemical elemental composition analysis (line, point and mapping). The SE and VPSE detectors enable to observe sample topography, depending on high vacuum (SE) or variable vacuum (VPSE) used during the research, whereas BSD detector allows a better contrast while observing the sample in both conditions, which is especially helpful during EDS analysis. Scanning electron microscopy with this device allows for testing the microstructure of materials of a maximum height of $100 \mathrm{~mm}$, a diameter up to $230 \mathrm{~mm}$ and weight not exceeding $0.5 \mathrm{~kg}$. The possible magnification enlarges the view from several dozen to a several dozens of thousands of times.

The pictures made by the author during the research show a sample of mortar after pre-cracking and after healing the crack with the polymer that has leaked from the capsule. The images were taken at a magnification of 50x, maintaining a working distance of $16 \mathrm{~mm}$. A BSD detector was used, and tests were carried out at accelerating voltage of $20 \mathrm{kV}$.
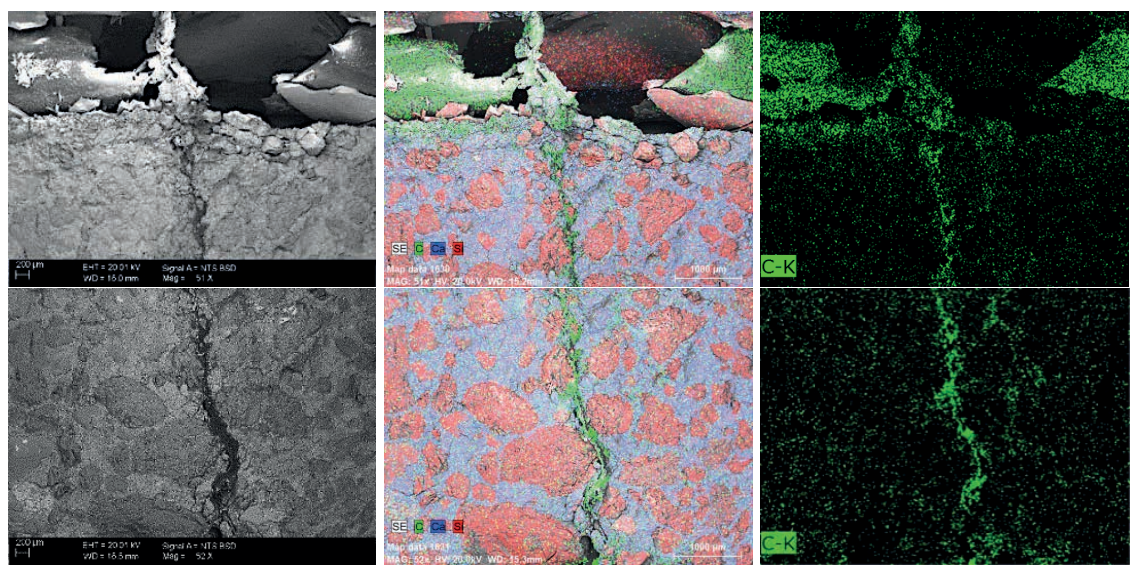

Fig. 3. SEM image of a part of capsule after the healing process, a visible crack filled with polymer, at 50x magnification. Surface distribution of chemical elements: carbon (green), calcium (blue) and silicon (red). Carbon mapping indicates the crack filled with polymer. The first line shows capsule and the second line the further part of sample along the crack. Source: the author's own study 


\subsection{The results of micro-strength test in scanning electron microscopy by using a tensile stage}

For the purpose of the research, vitamin (powder) micro-capsules in shape of oval were stuck with cyanoacrylate glue to previously prepared handles (printed with a $3 \mathrm{D}$ printer). Prepared research elements were sprayed with gold to obtain a better visual effect under microscope. Pictures of samples and tests results carried out with the use of a tensile stage are presented in Figure 4-5.
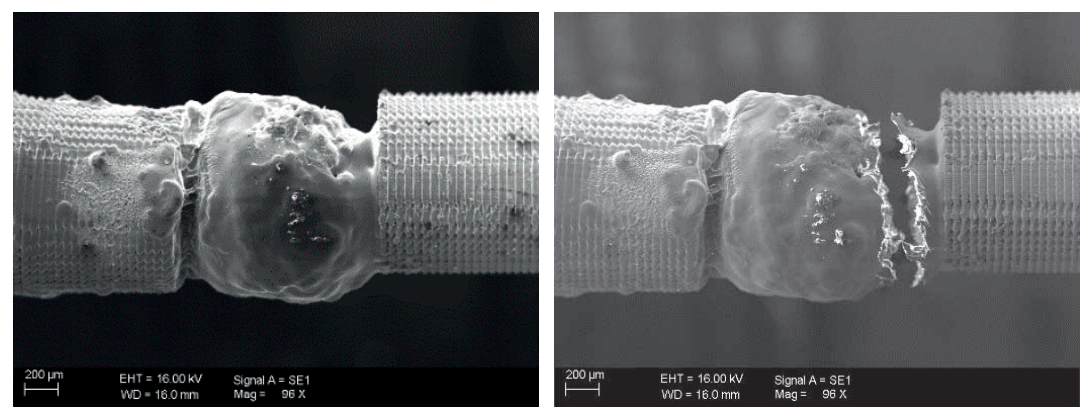

Fig. 4. SEM images of attached powder micro-capsule installed in a tensile stage (on the left) and micro-capsule after stretching (on the right). The test was performed at 96x magnification. Source: the author's own study

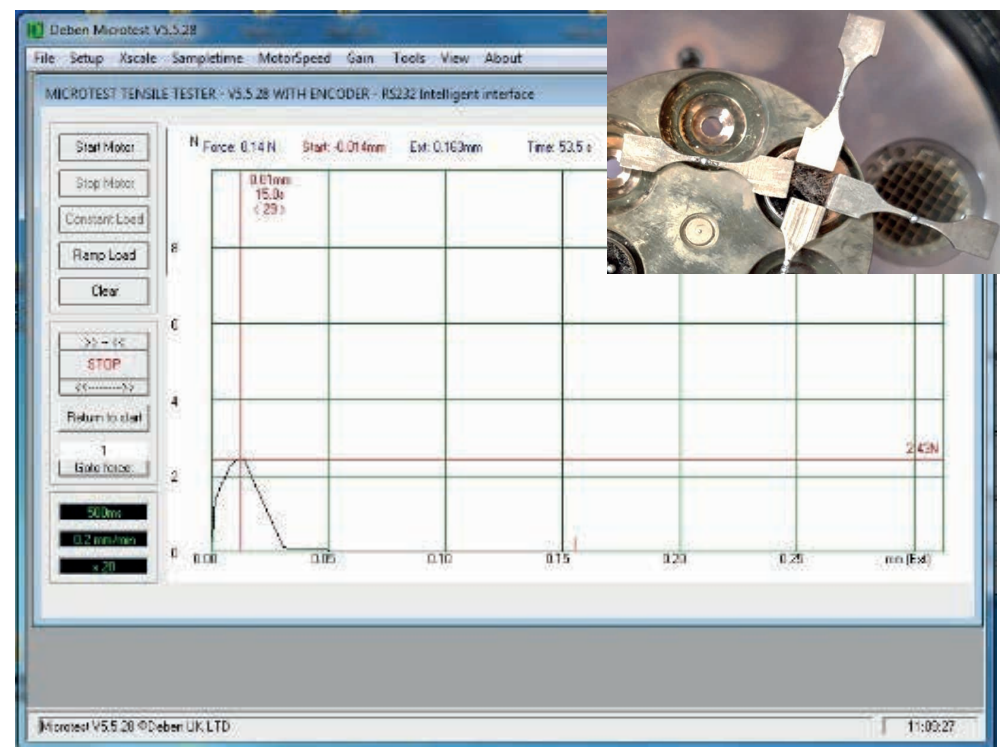

Fig. 5. The result of tensile strength test of a powder micro-capsule under a tensile stage mounted in the microscope chamber. Examination of the impact of the force and elongation of the sample. Photo of the sample with $2 \mathrm{~cm}$ long handles (micro-capsule attached to handles printed in a 3D printer). Source: the author's own study

The research allowed to determine the elongation of the sample and the maximum value of force needed to destroy the element. The video made during the test made it possible to recognize the moment when the first cracks appeared in the material. The destruction was 
not very rapid, with the time lag between consecutive cracks, and finally a part of material broke away from the capsule along the line of the cracks. A graph showing impact of force on elongation was obtained, due to which it was possible to determine the tensile strength of the capsule. The maximum force value was $2.43 \mathrm{~N}$ and elongation was $0.01 \mathrm{~mm}$, which gives approximately the strength of $3 \mathrm{MPa}$.

\section{Conclusions}

The results of the research described in the article are the basis for assessing the possibility of using an optical microscope and scanning electron microscope with a tensile stage for testing the process of self-healing of cementitious materials. The microscopic observation techniques are commonly used in tests and observations of the materials with self-healing properties. However, every type of material requires to adapt the research to the methodology. Other interesting feature is the possibility to determine micro-mechanical properties of engineering materials, such as other capsules.

In the research described in the paper, the observations were made on mortar beams modified with macro-capsules filled with polymer with the use of both optical and scanning microscopes. By using these research methods, it is possible to visually assess whether a given crack has been sealed. Research with the use of an optical microscope resulted in a preview of a larger part of the sample due to the larger field of view. Scanning microscopy, however, enabled more accurate observations of the crack and the polymer that filled it. The use of chemical analysis has facilitated the assessment of the effectiveness of the healing process. In this case, the polymer marked with the green marker shows exactly where the polymers were distributed in the crack.

The test with the use of a tensile stage was carried out on micro-capsules, in this case on prototype samples in order to better understand the capabilities of the device. It is crucial to determine mechanical strength of both micro- and macro-capsules in self-healing materials. These capsules should break when the material (mortar in this case) is pre-cracked, not before. They should not either be more durable than the material itself. The determination of their mechanical characteristics enables the selection of a suitable material for the carrier of the healing agent.

\section{References}

[1] Takagi T., "Present state and future of the intelligent materials and systems in Japan", Journal of Intelligent Material Systems and Structure, no. 10, (1999), pp. 575-581.

[2] „Polimery są materiałami przyszłości”, Chemia i Biznes., no. 3, (2015), Available: https://www. chemiaibiznes.com.pl/aktualnosc/polimery-sa-materialami-przyszlosci [Accessed: 25 Nov 2019]

[3] Zwaag S. (ed), "Self-healing materials, an alternative approach to 20 centuries of materials science", Springer Series in Materials Science, vol. 100, Springer, Dordrecht 2007.

[4] Hager M.D. et al., (2010). „Self-healing materials“, Advanced Materials, pp. 5424-543, https:// doi.org/10.1002/adma.201003036, quoted after: Zając B., Gołebiowska I., „Procesy odtwórcze w materiałach cementowych“, Inżynieria i Aparatura Chemiczna vol. 55, no. 3, (2016), pp.112-113.

[5] Rooij M. et al., State-of-the-Art Reports, Self-Healing Phenomenain Cement-Based Materials. Editors Technical Committee 221-SHC: Self-Healing Phenomenain Cement-Based Materials. Springer 2013.

[6] Tziviloglou E. et al., "Bio-based Self-healing Mortar: An Experimental and Numerical Study", Journal of Advanced Concrete Technology, vol. 15, no. 9, (2017), pp. 536-543. https://doi.org/10.3151/ jact.15.536 
[7] Alghamri R. Kanellopoulos A. Al-Tabbaa., "Impregnation and encapsulation of lightweight aggregates for self-healing concrete", Construction and Building Materials, no. 124, (2016), pp. 910-921. https://doi.org/10.1016/j.conbuildmat.2016.07.143

[8] Qureshi T.S., Kanellopoulos A., Al-Tabbaa., "Encapsulation of expansive powder minerals within a concentric glass capsule system for self-healing concrete", Construction and Building Materials, no. 121, (2016), pp. 629-643. https://doi.org/10.1016/j.conbuildmat.2016.06.030

[9] Al-Ansari M., et al., "Performance of modified self-healing concrete with calcium nitrate microencapsulation", Construction and Building Materials, no. 149, (2017), pp. 525-534. https://doi. org/10.1016/j.conbuildmat.2017.05.152

[10] Dong B. W. et al., "Smart releasing behavior of a chemical self-healing microcapsule in the stimulated concrete pore solution", Cement and Concrete Composites, no. 56, (2015), pp .46-50. https://doi.org/10.1016/j.cemconcomp.2014.10.006

[11] Yildirim G. et al., "A review of intrinsic self-healing capability of engineered cementitious composites: Recovery of transport and mechanical properties", Construction and Building Materials, no. 101, (2015), pp. 10-21. https://doi.org/10.1016/j.conbuildmat.2015.10.018 